\title{
Cesárea Electiva como Factor de Riesgo Asociado al Síndrome de Distress Respiratorio Neonatal
}

\section{Elective Cesarean Section as a Risk Factor Associated with Neonatal Respiratory Distress Syndrome}

Diana Mishelle Moncayo Rivera ${ }^{1}$, Christian Miguel Moncayo Rivera . ${ }^{2}$, Justa María Serpa Calderón. ${ }^{3} \&$ Nelson Ricardo Chacha Suscal. ${ }^{4}$

\begin{abstract}
Foundations: Elective Cesarean section is a surgical procedure that has increased worldwide in recent years. Respiratory morbidities are the most frequent complications in neonates born in this way, especially due to the absence of labor.

Objective: To determine, from a systematic literature review, elective

\section{Resumen}

Fundamentos: La cesárea electiva es un procedimiento quirúrgico que ha incrementado en todo el mundo en los últimos años. Las morbilidades respiratorias constituyen las complicaciones más frecuentes en neonatos nacidos por esta vía, en especial por la ausencia de trabajo de parto.

\footnotetext{
${ }^{1}$ Universidad Católica de Cuenca, Facultad de Medicina, Cuenca, Ecuador, email: diana.moncayo090997@gmail.com. https://orcid.org/0000-0002-3364-8052. .

2 Ministerio de salud pública, Cuenca, Hospital Vicente Corral Moscoso, christianmoncayo487@gmail.com. https://orcid.org/0000-0002-4734-7314

3 Ministerio de salud pública, Cuenca, Distrito de salud, justa0000@gmail.com, https://orcid.org/0000-0002-2099-5100

4 Ministerio de salud pública, Cuenca, Hospital Vicente Corral Moscoso, ricardocsmed93@gmail.com. https://orcid.org/0000-0002-2096-5100
} 
cesarean section as a risk factor for respiratory distress morbidity and mortality in newborns.

Methods: Fourteen articles were included on this topic that met certain inclusion criteria. The search was carried out in different journals such as Scielo, Cochrane, Medwave, Redalyc, PubMed, among others, published in the last 5 years, in English and Spanish language. Mendeley software was used to organize the information.

Results: Developing countries have rates of the elective cesarean section above $40 \%$, especially in the private sector. Neonatal respiratory morbidity-mortality increases by elective cesarean section being up to 3 times higher compared to vaginal delivery. Transient Tachypnea of the Newborn was the most frequent entity of Neonatal Respiratory Distress Syndrome.

Conclusions: Elective cesarean section is a problem in underdeveloped countries because it brings with it important fetal complications such as Transient Tachypnea of the Newborn, a pathology that can be prevented: firstly by properly educating the mother, adopting measures such as premature labor in patients with a history of previous cesarean section, the use of antenatal steroids and the administration of oxytocin.

Keywords: respiratory distress, elective cesarean section, neonate, Transient Tachypnea of the Newborn
Objetivo: Determinar, a partir de una revisión bibliográfica sistemática, la cesárea electiva como factor de riesgo para la morbi-mortalidad por distress respiratorio en recién nacidos.

Métodos: se incluyeron 14 artículos en relación a este tema los cuales cumplieron determinados criterios de inclusión. La búsqueda se realizó en diferentes revistas como Scielo, Cochrane, Medwave, Redalyc, PubMed, entre otros, publicadas en los últimos 5 años, en idioma inglés y español. Se empleó el software Mendeley para la organización de la información.

Resultados: los países subdesarrollados presentan tasas de cesáreas electivas superiores al $40 \%$, en especial en el sector privado. La morbi-mortalidad respiratoria neonatal aumenta por cesárea electiva siendo hasta 3 veces superior a comparación del parto vaginal. La Taquipnea Transitoria del Recién Nacido fue la entidad más frecuente del Síndrome de Distress Respiratorio Neonatal.

Conclusiones: la cesárea electiva constituye una problemática en países subdesarrollados ya que trae consigo complicaciones fetales importantes como la Taquipnea Transitoria del Recién Nacido, patología que se puede prevenir: en primera instancia educando adecuadamente a la madre, la adopción de medidas como el trabajo de parto previo en pacientes con antecedentes de cesárea anterior, el uso de corticoides antenatales y la administración de oxitocina. 
Palabras Clave: Distress Respiratorio, Cesárea Electiva, Neonato, Taquipnea

Transitoria del Recién Nacido.

\section{Introducción}

La Organización Mundial de la Salud (OMS), recomienda que la tasa de nacimientos por cesárea en un país debe encontrarse entre el 5 y 15\% del total (Martínez, 2015), pero en América Latina para el año 2016, se incrementó hasta un 38.9\% (Bernal, 2018).

Aquello indica que ha ocurrido un aumento desproporcionado de cesáreas que probablemente no son necesarias, realizadas sin indicación médica y propuestas a las pacientes como forma de parto más seguro, es decir, un reemplazo del proceso natural del nacimiento por una intervención quirúrgica (Molina, 2019).

Las morbilidades respiratorias se informan como las complicaciones más frecuentes asociadas con los partos por cesárea. En un estudio realizado en el Hospital Universitario de Guayaquil efectuado en el año 2015, Hidalgo concluye que el $73 \%$ de neonatos nacidos por cesárea programada presentó Síndrome de Distress Respiratorio.

Esta patología se define como los síntomas clínicos de la dificultad respiratoria neonatal temprana con características radiológicas de tórax consistentes y que además requiere suplementación de oxígeno dentro de las 24 horas posteriores al nacimiento para mantener una saturación de oxígeno óptima. Continúa siendo la causa principal de mortalidad y morbilidad tempranas durante la infancia y la niñez, que afecta aproximadamente al $1 \%$ de los recién nacidos (Li et al., 2019).

El riesgo de sufrir Distrés Respiratorio guarda estrecha relación con la vía de parto. El motivo reside durante el inicio del trabajo de parto: el feto sufre una situación de estrés ya que sus pulmones intentan expulsar el líquido residual por su boca para llevar a cabo la primera respiración lo cual se ve favorecido por las contracciones uterinas y los movimientos realizados por el feto en el canal vaginal; al no existir este mecanismo en las cesáreas programadas la susceptibilidad de presentar complicaciones respiratorias incrementa hasta cuatro veces más un recién nacido a comparación del parto vaginal. Además, el riesgo aumenta alrededor de 1,5 veces por cada semana si la cirugía ocurre antes de las 39 semanas de gestación.

Este estudio se plantea con el objetivo de determinar, a través de la comparación de múltiples estudios científicos, el parto por cesárea electiva como factor de riesgo para la morbi mortalidad por distrés respiratorio en recién nacidos, de tal manera se podrá conocer la frecuencia, identificar la asociación estadística entre cesárea electiva (el factor de riesgo de estudio) y el distrés respiratorio y las principales patologías asociadas al mismo. 
Por esta razón se pretende revertir dicho incremento, para de tal manera disminuir el índice de frecuencia de la operación cesárea tanto en el sector público como en el privado, bajo indicación médica de forma indispensable, obteniendo como consiguiente menor costo a nivel hospitalario, una mejor atención en el trabajo de parto de la gestante y, por lo tanto, mejor beneficio materno-fetal (\& Wolfman, 2015).

\section{Metodologia}

Diseño: Se realizó una revisión sistemática de fuentes bibliográficas que realizaron el estudio: "CESÁREA ELECTIVA COMO FACTOR DE RIESGO ASOCIADO AL SÍNDROME DE DISTRESS RESPIRATORIO NEONATAL”. Para la localización de la información se emplearon palabras claves como Distrés Respiratorio, Cesárea Electiva, Neonato, Evidencia Científica, Labor de Parto, Parto Vaginal.

Estrategia de Búsqueda: se efectuó una búsqueda general en la Biblioteca Virtual de la Universidad Católica de Cuenca y en Google Académico lo cual arrojó alrededor de 2152 resultados 2101 y 51 respectivamente, que incluían revistas científicas de distintos sitios web en Ecuador y a nivel internacional empleando palabras claves como Distrés Respiratorio, Cesárea Electiva, Neonato, Evidencia Científica, Labor de Parto, Parto Vaginal.

Posteriormente se ejecutó una exploración en la base de datos de Scielo, PubMed, Medline, Cochrane, Elsevier, entre otros, para la obtención documentos que incluyeron las palabras claves antes mencionadas y sus combinaciones, con publicaciones de los últimos 5 años en idiomas como español e inglés excluyendo 2040; 61 documentos fueron sometidos a lectura de texto completo.

Tras la lectura se excluyeron 47, incluyendo finalmente 14 documentos. Lo antes descrito se resume en el gráfico $\mathrm{N}^{\circ} 1$.

\section{Criterios de Inclusión}

Artículos que correspondieron en título y resumen, centrando a los conceptos de distrés respiratorio y partos por cesárea programada, cuyas publicaciones se realizaron entre los años 2015 al 2020 en idiomas de inglés y español.

\section{Criterios de Exclusión}

Se descartó todo aquel estudio que no presente resultados concluyentes, y que el análisis del estudio no estuvo regido al ámbito de la investigación o que se aprecie la observación de otras profesiones que no son coherentes acerca del contexto del parto por cesárea como factor de riesgo del distrés respiratorio en neonatos. 
Revisiones sistemáticas y artículos científicos relacionados con la cesárea electiva como factor de riesgo asociado al distrés respiratorio neonatal no correspondientes a los últimos cinco años.

Documentos redactados en idiomas distintos al inglés y español.

Organización de la información: Con ayuda del programa Mendeley, se procedió a la selección de documentos de la literatura gris, revisiones sistemáticas, artículos científicos.

Se obtuvo en la búsqueda inicial alrededor de 2152 artículos, se excluyeron 2138 que no fueron relevantes para el objetivo de esta revisión, para finalmente seleccionar 14 artículos.

Análisis de los datos: Una vez seleccionados los documentos científicos con ayuda del programa Mendeley se procedió a la extracción de información sobre autoría, año, fuentes de información.

\section{Resultados}

La cesárea electiva es un procedimiento que se realiza de forma programada antes de un trabajo de parto verdadero realizado en gestantes con patologías en donde no se recomiende un parto vaginal (Maternofetal, 2020; MSP, 2015).

Se ha visto un incremento masivo de cesáreas programadas en las últimas décadas, como es el caso del Reino Unido y Estados Unidos con porcentajes mayores al 30\%, Brasil con más del $40 \%$ al igual que México, Paraguay y Ecuador, las cuales son cifras alarmantes en comparación de la recomendación de la media $10 \%$ por país establecido por la Organización mundial de la Salud (Bernal, 2018).

Sin embargo, sus ventajas no justifican su aumento continuo ya que conlleva considerables complicaciones respiratorias neonatales, en comparación con el parto vaginal (Benzouina et al., 2016).

En el Hospital de Ventanilla-Perú durante el año 2016, se comparó 197 neonatos nacidos por parto vaginal y cesárea: 48,22\% tuvieron Síndrome de Distrés Respiratorio Neonatal (SDRN) de los cuales se encontró que aquellos nacidos por cesárea electiva tuvieron un porcentaje de 41,6, además se describieron como factores de riesgo el sexo masculino y macrosomía fetal (Trujillo Angeles, 2018). Similar resultado se obtuvo en el trabajo realizado por Bautista (2018) en el Hospital Vicente Corral Moscoso-Ecuador donde el porcentaje de distrés respiratorio en recién nacidos por cesárea electiva fue superior al $60 \%$, y su coterráneo Hidalgo (2015) en el Hospital Universitario de Guayaquil que describió 77 pacientes ingresados a la unidad de cuidados intensivos neonatales con diagnóstico de SDRN de los cuales el 73\% nacieron por cesárea electiva. Por el contrario, Pirjani (2018) establece que no existe diferencia entre las complicaciones neonatales frecuentes como sepsis neonatal o SDRN 
El mecanismo principal de las complicaciones respiratorias en los neonatos nacidos por cesárea programada reside en la ausencia de trabajo de parto; esto es, el líquido pulmonar residual que el feto intenta expulsar por su nariz y boca, dado por las contracciones uterinas y movimientos en el canal vaginal, no se llevan a cabo, por lo que el feto presenta dificultad respiratoria al nacer.

En el estudio analítico realizado en 25 Centros Obstétricos de Estados Unidos, con alrededor de 63180 mujeres incluidas, se intentó un trabajo de parto previo a su cesárea electiva en donde el $18 \%$ presentó distrés respiratorio y se concluyó como un factor de protección para el recién nacido. Así mismo, en el Hospital Núñez de Perú, se analizaron más de 160 pacientes en las que se realizó un trabajo de parto previo a la cesárea programada, en donde la frecuencia de distrés respiratorio fue inferior al 30\% comparado con aquellas en donde no se les practicó el trabajo de parto por lo que se estableció como un factor de riesgo.

La variante más frecuente del Síndrome de Distress Respiratorio se describe como la Taquipnea Transitoria del Recién Nacido (TTRN), patología que se da principalmente en neonatos a término por retención de líquido a nivel pulmonar que normalmente se extrae por las contracciones producidas por la madre en el parto vaginal (Guia Práctica Clinica, 2016; Mancilla \& Villanueva, 2016). Shukla \& Mittal (2015) lo comprueban en su estudio retrospectivo realizado en el año 2015 en Karnataka-India, donde de un total de 134 neonatos nacidos por cesárea electiva, la incidencia de TTRN fue del 15,8\% y del 6,3\% en los recién nacidos de 37 a 38, 6 y $\geq 39$ semanas de gestación respectivamente.

Se señala que el índice de cesáreas es mayor a nivel del sector privado que en el público, en especial por petición materna, principalmente por sugerencia por parte del médico, 38,3\% de las cesáreas electivas en una revisión realizada en Irán (Rafiei \& Ghare Naz, 2018).

Es así que la investigación realizada en México aproximadamente el 63\% fue realizado de forma privada (C. Garcíal, 2018), en Brasil cerca de un 72\%, y en China , la tasa de cesáreas electivas en sectores privados es mayor al 71\%. (Rosenberg \& Trevathan, 2018), sin embargo, en Chile, cerca del 77,8\% de la población cree que es de menos riesgo el parto vaginal que la cesárea (Carvajal C \& Ralph T, 2018).

Elementos como la administración de oxitocina y corticoide antes de la realización de una cesárea programada, se describen como factores protectores, lo que se indica en la revisión sistemática realizada en el Hospital del Reino Unido por Gazitúa y su colega Pérez, además del trabajo de parto, en donde la frecuencia de distrés respiratorio fue inferior al $25 \%$.

$\mathrm{Al}$ ser un procedimiento quirúrgico, se requiere anestesia que podría ser general o raquídea según sea el caso; se verifica que en la anestesia espinal (la más empleada) hay muy pocas posibilidades de intoxicación farmacéutica y menor cantidad de fármaco se transfiere al feto y no se ha establecido complicaciones respiratorias fetales (Abdollahpour et al., 2015). 
Los resultados del estudio se resumen en la Tabla $\mathrm{N}^{\mathrm{o}} 1$ :

Tabla №1.- Estudios relacionados con la cesárea electiva como factor de riesgo para el desarrollo de Distress Respiratorio Neonatal.

\begin{tabular}{|c|c|c|c|c|c|}
\hline $\begin{array}{l}\text { REFER. } \\
\text { BIBLIO. }\end{array}$ & AUTOR & AÑO & FINALIDAD & FUENTE & CONCLUSIONES \\
\hline $\begin{array}{l}\text { (Hidalgo } \\
\text {, 2015) }\end{array}$ & $\begin{array}{l}\text { Hidalgo, } \\
\text { A. }\end{array}$ & 2015 & $\begin{array}{lr}\begin{array}{l}\text { Determinar } \\
\text { factores }\end{array} & \text { los } \\
\text { riesgo que } & \text { se } \\
\text { asocian } & \text { al } \\
\text { Síndrome } & \text { de } \\
\text { dificultad } & \\
\text { respiratoria } & \text { en } \\
\text { nacimientos } & \text { por } \\
\text { cesárea o vía } \\
\text { vaginal. }\end{array}$ & $\begin{array}{lr}\text { Estudio analítico, } \\
\text { retrospectivo de } \\
\text { casos y controles } \\
\text { en el Hospital } \\
\text { Universitario de } \\
\text { Guayaquil, } & 77 \\
\text { pacientes } & \text { con } \\
\text { diagnóstico } & \text { de } \\
\text { distrés } & \\
\text { respiratorio } & \\
\text { neonatal. }\end{array}$ & $\begin{array}{l}\text { Se establece que la } \\
\text { mortalidad y morbilidad } \\
\text { neonatal del Ecuador } \\
\text { posee un riesgo de } 2,5 \\
\text { en recién nacidos por } \\
\text { cesárea electiva. }\end{array}$ \\
\hline $\begin{array}{l}\text { (Bautist } \\
\text { a, 2018) }\end{array}$ & $\begin{array}{l}\text { Bautista } \\
\text {, L. \& } \\
\text { Izquierd } \\
\text { o, C. }\end{array}$ & 2018 & $\begin{array}{lr}\text { Conocer la } & \text { la } \\
\text { prevalencia } & \text { de } \\
\text { un tipo de SDRN } \\
\text { (TTRN) } \\
\text { neonatos } \\
\text { nacidos por } \\
\text { cesárea y parto } \\
\text { vaginal. }\end{array}$ & $\begin{array}{l}\text { Estudio analítico, } \\
\text { casos y controles, } \\
\text { retrospectivo } \\
\text { realizado en el } \\
\text { Hospital Vicente } \\
\text { Corral Moscoso, } \\
\text { retrospectivo en } \\
\text { donde } \\
\text { participaron } 834 \\
\text { neonatos, nacidos } \\
\text { por cesárea y } \\
\text { parto vaginal. }\end{array}$ & $\begin{array}{l}\text { La frecuencia de TTRN } \\
\text { es superior a } \\
\text { comparación de la } \\
\text { bibliografía analizada, se } \\
\text { encontraron factores de } \\
\text { riesgo como la cesárea } \\
\text { electiva. }\end{array}$ \\
\hline $\begin{array}{l}\text { (Trujillo } \\
\text { Angeles } \\
\text {, 2018) }\end{array}$ & $\begin{array}{l}\text { Trujillo, } \\
\text { W. }\end{array}$ & 2018 & $\begin{array}{lr}\text { Describir } & \text { los } \\
\text { factores } & \text { de } \\
\text { riesgo que } & \text { se } \\
\text { asocian } & \text { al } \\
\text { SDRN } & \text { en } \\
\text { nacimientos } & \text { por } \\
\text { cesárea o vía } \\
\text { vaginal. }\end{array}$ & $\begin{array}{l}\text { Estudio analítico, } \\
\text { retrospectivo de } \\
\text { casos y controles } \\
\text { en el Hospital de } \\
\text { Ventanilla de } 197 \\
\text { neonatos nacidos } \\
\text { por cesárea } \\
\text { programadas y } \\
\text { parto vaginal. }\end{array}$ & $\begin{array}{l}\text { Los factores que se } \\
\text { relacionaron al } \\
\text { desarrollo de TTRN } \\
\text { fueron principalmente la } \\
\text { cesárea electiva, género } \\
\text { masculino y macrosomía } \\
\text { fetal. }\end{array}$ \\
\hline
\end{tabular}




\begin{tabular}{|c|c|c|c|c|c|}
\hline $\begin{array}{l}\text { (Morale } \\
\text { s } \\
\text { Máximo } \\
\& \\
\text { Morales } \\
\text { Máximo, } \\
\text { 2016) }\end{array}$ & $\begin{array}{l}\text { Morales, } \\
\text { D. }\end{array}$ & 2015 & $\begin{array}{l}\text { Conocer las } \\
\text { complicaciones } \\
\text { tanto maternas } \\
\text { como fetales de } \\
\text { la cesárea } \\
\text { electiva en } \\
\text { comparación } \\
\text { con el parto } \\
\text { vaginal }\end{array}$ & $\begin{array}{l}\text { Se realizó un } \\
\text { estudio } \\
\text { descriptivo, tipo } \\
\text { comparativo en } \\
\text { donde se trabajó } \\
\text { con } 150 \text { gestantes } \\
\text { sometidas a } \\
\text { cesárea electiva y } \\
\text { parto vaginal, y su } \\
\text { relación con el } \\
\text { desarrollo de } \\
\text { morbilidad } \\
\text { respiratoria } \\
\text { neonatal. }\end{array}$ & $\begin{array}{l}\text { Se concluyó que ambas } \\
\text { vías, presentan riesgos } \\
\text { similares de distrés } \\
\text { respiratorio y la decisión } \\
\text { de su realización } \\
\text { depende de la evolución } \\
\text { de la madre y el neonato, } \\
\text { todo ello en base a la } \\
\text { decisión del médico. }\end{array}$ \\
\hline $\begin{array}{l}\text { (Césped } \\
\text { es, } \\
2016 \text { ) }\end{array}$ & $\begin{array}{l}\text { Césped } \\
\text { es, A. }\end{array}$ & 2016 & $\begin{array}{l}\text { Indicar la } \\
\text { complicación } \\
\text { SDRN en la } \\
\text { cesárea electiva } \\
\text { y el parto vaginal }\end{array}$ & $\begin{array}{lr}\text { Estudio analítico } \\
\text { en el } & \text { Hospital } \\
\text { Rafael } & \text { A. } \\
\text { Calderón, } & 396 \\
\text { pacientes } & \\
\text { sometidas } & \text { a } \\
\text { cesárea electiva y } \\
1188 \text { a parto } \\
\text { vaginal. }\end{array}$ & $\begin{array}{l}\text { Un embarazo >39 } \\
\text { semanas o a término } \\
\text { sometido a parto vaginal } \\
\text { es menos riesgoso que } \\
\text { el parto vaginal, en } \\
\text { especial en cuanto a } \\
\text { morbilidad respiratoria. }\end{array}$ \\
\hline $\begin{array}{l}\text { (Nakimu } \\
\text { li \& } \\
\text { Nakubul } \\
\text { wa, } \\
2015)\end{array}$ & $\begin{array}{l}\text { Nakimuli } \\
\text { Et al }\end{array}$ & 2015 & $\begin{array}{l}\text { Establecer la } \\
\text { incidencia y } \\
\text { determinantes } \\
\text { de la morbilidad } \\
\text { neonatal de la } \\
\text { cesárea electiva }\end{array}$ & $\begin{array}{l}\text { Estudio analítico, } \\
\text { retrospectivo: } \\
25.846 \text { cesáreas } \\
\text { electivas en el } \\
\text { Hospital de } \\
\text { Mulago. }\end{array}$ & 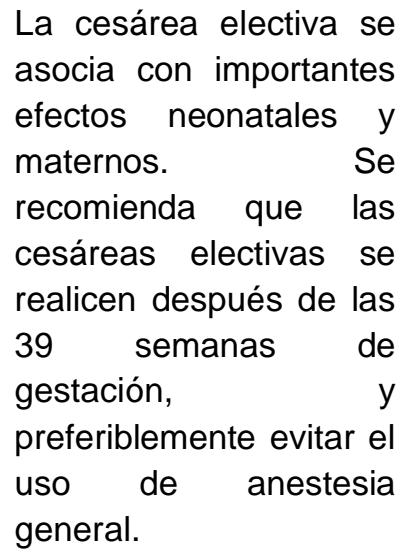 \\
\hline $\begin{array}{l}\text { (Li et al., } \\
\text { 2019) }\end{array}$ & $\begin{array}{l}\text { Zhang, } \\
\text { C., } \\
\text { Zhang, } \\
\text { D., \& Li, } \\
\text { Y. }\end{array}$ & 2019 & $\begin{array}{l}\text { Describir la } \\
\text { asociación de la } \\
\text { cesárea electiva } \\
\text { y el desarrollo de } \\
\text { dificultad } \\
\text { respiratoria } \\
\text { neonatal }\end{array}$ & $\begin{array}{l}\text { Meta-análisis de } \\
15 \text { estudios } \\
\text { relacionados a la } \\
\text { asociación entre } \\
\text { cesárea electiva y } \\
\text { el riesgo de } \\
\text { neonatal } \\
\text { distrés } \\
\text { respiratorio: } \\
\text { estudios } \\
\text { América en } \\
\end{array}$ & $\begin{array}{l}\text { El desarrollo de dificultad } \\
\text { respiratoria neonatal } \\
\text { está íntimamente } \\
\text { desarrollado con la } \\
\text { cesárea que se realiza } \\
\text { de forma programada } \\
\text { con una probabilidad } \\
\text { hasta de } 2,8 \text {. }\end{array}$ \\
\hline
\end{tabular}


Norte, 6 estudios

en Europa y uno

en Asia

(Benjam Beth, A. 2019 Asociación del Se seleccionaron

in M. Et al

Davis,

Glen F.

Rall,

2017)

(Abdela Abdelazi 2017

zim et $\mathrm{mEt}$ al

al.,

2017) trabajo de parto

con

producción de

morbilidad

respiratoria en

neonatos a

término

\section{Hospitales de}

EE. UU., 63.187

gestantes y 10629

sometidas a labor

de parto

a

(1)

\section{Conocer el 965 recién La morbilidad impacto de la nacidos de $>=37$ respiratoria neonatal administración semanas de asociada con la cesárea de oxitocina gestación nacidos electiva disminuyó antenatal en la por cesárea significativamente generación de electiva, en dos después de la morbilidad grupos: expuestos exposición prenatal a la respiratoria en y no expuestos a oxitocina. Una reducción neonatos oxitocina. significativa \\ asociado con la cesárea \\ electiva. \\ de morbilidad respiratoria neonatal se lograría si se realizara cesárea electiva \\ después de 39 semanas de gestación.}

\begin{tabular}{|c|c|c|c|c|c|}
\hline $\begin{array}{l}\text { (Dileep } \\
\text { et al., } \\
2015 \text { ) }\end{array}$ & $\begin{array}{l}\text { Dileep, } \\
\text { A., } \\
\text { Babar, } \\
\text { N., } \\
\text { Sadiq, } \\
\text { S. }\end{array}$ & 2015 & $\begin{array}{ll}\text { Comparar la } & \text { la } \\
\text { morbilidad } & \\
\text { respiratoria } & \\
\text { neonatal en la } \\
\text { cesárea } & \\
\text { electiva. } & \end{array}$ & $\begin{array}{l}\text { Estudio } \\
\text { retrospectivo en el } \\
\text { Hospital de } \\
\text { Karachi en base al } \\
\text { historial médico } \\
\text { de gestantes } \\
\text { sometidas a } \\
\text { cesárea electiva, } \\
196 \text { respectivamente, } \\
\text { con y sin } \\
\text { administración de } \\
\text { dexametasona } \\
\text { antenatal. }\end{array}$ & $\begin{array}{l}\text { Se encontraron efectos } \\
\text { beneficiosos de la } \\
\text { dexametasona } \\
\text { profiláctica en la } \\
\text { morbilidad respiratoria } \\
\text { neonatal, pero se } \\
\text { requieren más estudios } \\
\text { prospectivos con un gran } \\
\text { tamaño de muestra. }\end{array}$ \\
\hline
\end{tabular}


ISSN: 2697-3391

Vol. 4, $\mathrm{N}^{\circ} 1$, p. 225-243, enero-marzo, 2021

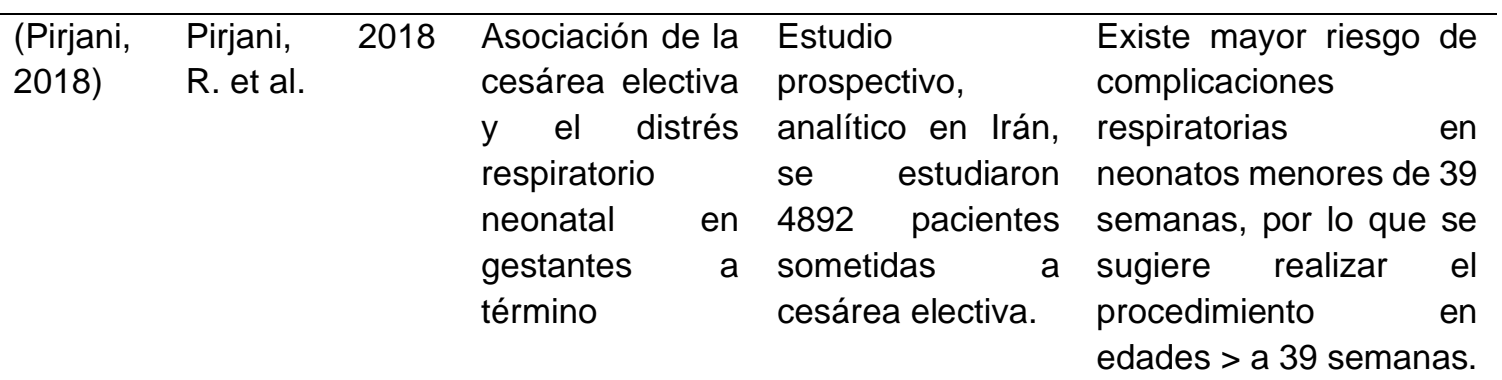

\begin{tabular}{|c|c|c|c|c|c|}
\hline $\begin{array}{l}\text { (Petour } \\
\text { Gazitúa, } \\
\text { 2015) }\end{array}$ & $\begin{array}{l}\text { Gazitúa, } \\
\text { F. \& } \\
\text { Pérez, J. }\end{array}$ & 2015 & $\begin{array}{l}\text { Uso de } \\
\text { corticoides } \\
\text { antes del parto } \\
\text { para la } \\
\text { reducción de la } \\
\text { morbilidad } \\
\text { respiratoria en } \\
\text { cesárea electiva }\end{array}$ & $\begin{array}{ll}\text { Se seleccionaron } \\
2 \text { estudios, en } \\
\text { donde } & \text { se } \\
\text { administró } & \\
\text { corticoide } & \\
\text { antenatal } & \end{array}$ & $\begin{array}{l}\text { El uso de corticoides en } \\
\text { cesáreas programadas } \\
\text { en neonatos a término } \\
\text { reduce la frecuencia de } \\
\text { SDRN. }\end{array}$ \\
\hline $\begin{array}{l}\text { (Shradd } \\
\text { ha K. } \\
\text { Shetty, } \\
2015 \text { ) }\end{array}$ & $\begin{array}{l}\text { Shraddh } \\
\text { a, S. \& } \\
\text { Shetty, } \\
\text { A. }\end{array}$ & 2015 & $\begin{array}{l}\text { Asociar la } \\
\text { aparición } \\
\text { morbilidad } \\
\text { neonatal con la } \\
\text { cesárea } \\
\text { electiva. }\end{array}$ & $\begin{array}{l}\text { Estudio } \\
\text { retrospectivo en el } \\
\text { Departamento de } \\
\text { G-O de } \\
\text { Karnataka-India, } \\
1739 \text { nacimientos } \\
\text { por vía vaginal y } \\
1151 \text { por cesárea } \\
\text { electiva. }\end{array}$ & $\begin{array}{l}\text { Los recién nacidos por } \\
\text { cesárea electiva a las } 37 \\
\text { a } 38,6 \text { semanas de } \\
\text { gestación tienen un } \\
\text { mayor riesgo de } \\
\text { desarrollar morbilidad } \\
\text { respiratoria a } \\
\text { comparación con los } \\
\text { niños nacidos después } \\
\text { de las } 39 \text { semanas. La } \\
\text { morbilidad respiratoria } \\
\text { puede ser } \\
\text { reducido al retrasar la } \\
\text { cesárea electiva hasta } \\
\text { las } 39 \text { semanas de } \\
\text { gestación. }\end{array}$ \\
\hline
\end{tabular}


ISSN: 2697-3391

Vol. 4, $\mathrm{N}^{\circ} 1$, p. 225-243, enero-marzo, 2021

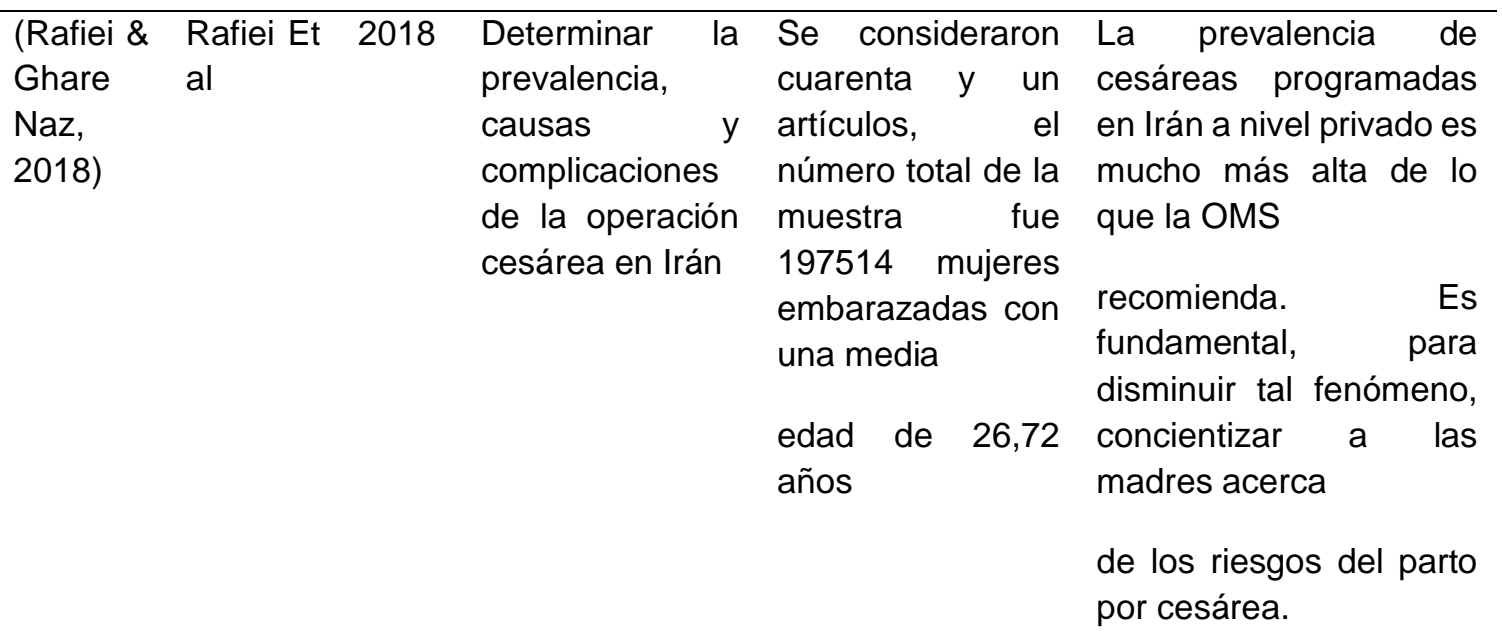

Fuente: Bibliografías consultadas

Elaborado por: Propia Autoría

\section{DISCUSIÓN}

14 artículos fueron considerados con respecto a ciertos criterios y se incluyeron en la revisión sistemática para realizar un estudio comparativo de la siguiente forma:

Gráfico №1.- Estrategia de búsqueda de revistas bibliográficas.

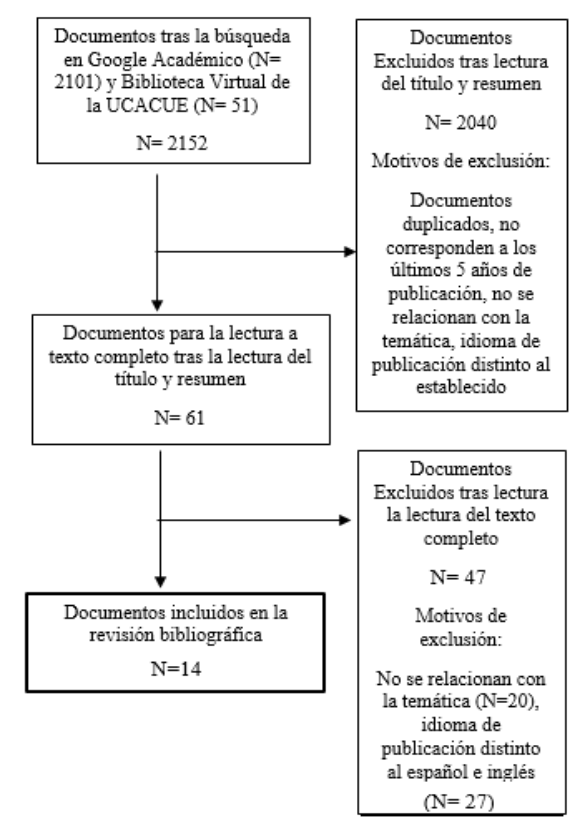

Elaborado por: Propia Autoría 
A nivel micro podemos basarnos en 2 estudios analíticos, retrospectivos de casos y controles realizados a nivel Nacional, Cuenca y Guayaquil: se encontró que la cesárea electiva se asocia de manera significativa con el desarrollo de Síndrome de Distress Respiratorio en el neonato, presentando hasta 2,7 y 2,4 veces más (valor de p inferior a 0,05), respectivamente, de probabilidad a comparación del parto vaginal (Bautista, 2018; Hidalgo, 2015).

Países latinoamericanos como Perú concuerdan con los anteriores enunciados. 2 estudios analíticos, retrospectivos de casos y controles: el primero incluyó 197 neonatos nacidos en edades gestacionales mayores a las 39 semanas por cesárea programada, 48,2\% presentaron taquipnea transitoria del recién nacido con un OR de 4.1 y además se observó mayor predisposición en el sexo masculino con un OR (razón de momios) de 8.1 (Trujillo Angeles, 2018). No obstante, el segundo estudio incluyó 150 pacientes sometidas a cesárea electiva, más del 13 por ciento presentaron complicaciones respiratorias como taquipnea transitoria del recién nacido (68\%), enfermedad de membrana hialina (32\%), pero el $12 \%$ de nacidos por parto vaginal también desarrollaron complicaciones respiratorias, por lo que establece que ambas vías de parto presentan el mismo riesgo (Morales Máximo \& Morales Máximo, 2016).

En Costa Rica, el estudio retrospectivo, analítico de cohorte transversal relata similitud en el desarrollo de dificultad respiratoria en neonatos nacidos por cesárea programada; trescientas noventa y seis pacientes expuestas al factor de riesgo, el $9.6 \%$ de neonatos presentaron distrés respiratorio con un riesgo relativo de 2.5 (valor de p de 0.05 ), igual que lo descrito en Ecuador (Céspedes, 2016).

En Uganda, país de África, se demostró en una investigación prospectiva similar a las anteriores, 116 recién nacidos por cesárea electiva de un total 5760 partos, quienes presentaron complicaciones neonatales, el 58\% fue por dificultad respiratoria con un OR de 3.01 y valor de P de 0.035 (Nakimuli \& Nakubulwa, 2015).

Un meta-análisis realizado en el año 2019, evaluaron 15 estudios relacionados a la asociación entre cesárea electiva y el riesgo de neonatal de distrés respiratorio, se realizaron 8 estudios en América del Norte, 6 estudios en Europa y uno en Asia. En cuanto al diseño del estudio, hubo 11 estudios de cohorte y 4 estudios de casos y controles. El OR combinado del riesgo de SDR neonatal asociado con cesárea electiva fue de 2.38, valor de P inferior a 0.05 (Li et al., 2019).

El mecanismo principal es la ausencia de un trabajo de parto verdadero. En el análisis realizado en distintos centros obstétricos de Estados Unidos, donde participaron 10560 gestantes, $2 \%$ (21 pacientes) en quienes se realizó cesárea programada por diagnóstico de cesárea anterior: en 6 pacientes se probó trabajo de parto previo al acto quirúrgico y el 10\% de neonatos presentaron distrés respiratorio, en comparación del 100\% de neonatos en donde no se realizó un trabajo de parto, presentando un OR de 2.91 (valor de P de 0.032), es decir, 
la labor de parto es un factor protector para el desarrollo de distrés respiratorio en neonatos nacidos por cesárea programada (Benjamin M. Davis, Glen F. Rall, 2017).

Un estudio observacional, retrospectivo realizado en Kuwait- País del Medio Oriente, se analizaron 65 neonatos nacidos por cesárea electiva que presentaron Taquipnea Transitoria del Recién Nacido (TTRN), en cuyas madres se administró oxitocina: el índice de difícultad respiratoria fue mayor en aquellas en donde no se administró la hormona (8 veces más de probabilidad de presentar morbilidad respiratoria) (Abdelazim et al., 2017).

Otro factor descrito como protector para evitar el desarrollo del Síndrome de Dificultad Respiratoria son los corticoides antenatales detallado en el artículo realizado en el Hospital de Pakistán. Se incluyeron 196 neonatos nacidos por cesárea programada; el 6\% presentó taquipnea transitoria del recién nacido, el $10 \%$ en neonatos en los que se administró corticoides antenatales 24 horas previos, y el $90 \%$ de los que no se les administró, por lo tanto, los neonatos que no son expuestos a dosis de corticoide antes de su nacimiento tienen un riesgo de 4,2 veces más de tener dificultad respiratoria en comparación de los expuestos al factor protector (valor de P de 0.05) (Dileep et al., 2015).

La cesárea programada es un procedimiento que se debe realizar según normas y protocolos, y si se efectúa en condiciones óptimas es considerado como seguro, pero si se realiza sin indicación médica que se justifique se obtendría mayores riesgos que beneficios (María \& Alonso, 2015; Schmitz et al., 2017). En países como México, en donde se observó que el mayor índice de cesáreas electivas se realizó de forma privada por petición materna, y el riesgo neonatal de padecer dificultad respiratoria es fue de 3 veces más a comparación de países desarrollados como China y Estados Unidos.

Se ha registrado un mayor número de casos de TTRN en el sector público en países como Ecuador ya que en su mayoría se realiza a partir de las 39 semanas de gestación, según lo establecido en el Ministerio de Salud (Bautista, 2018; Hidalgo, 2015; MSP, 2015).

En la investigación prospectiva de casos y controles realizado en Irán, se estudiaron 4892 pacientes sometidas a cesárea electiva, 2086 de los neonatos presentaron dificultad respiratoria con un OR de 2.9 (valor de p de 0.032 ), en donde el 52\% oscilaban en edades gestacionales entre 38 a 38.6 y el $48 \%$ de 39 semanas, a su vez el $76 \%$ del total presentó TTRN y el 24\% presentó Enfermedad de Membrana Hialina (Pirjani, 2018).

De igual forma, en la revisión sistemática de 452 mujeres con intervención por cesárea programada, en la ciudad de Suez de Egipto, se evidenció que el 23.2\% tuvo distrés respiratorio de los cuales el $19.6 \%$ con diagnóstico de taquipnea transitoria del recién nacidos en menores de 39 semanas, Riesgo relativo de 1.4 y valor de P de 0.02 (Petour Gazitúa, 2015). Similar resultado se obtuvo en la India, Shraddha \& Anil establecieron que existe un riesgo de 2.82 de probabilidad de que edades gestacionales entre 37 y 38 semanas presenten distrés respiratorio neonatal con su variante más común la TTRN, valor de P de menor a 0.05 . 
Por el contrario, Rafiei \& Ghare Naz (2018) afirman en su estudio que el motivo principal del incremento de cesáreas es a nivel privado, por sugerencia médica y al realizarse antes de las 38 semanas de gestación, son prevalentes patologías como la Enfermedad de Membrana Hialina en un 20\% superior a la TTRN e inferior a un 5\% al Síndrome de Aspiración Meconial

Por lo tanto, se sugiere la administración de corticoides antenatales, probar el trabajo de parto en pacientes con cesáreas anteriores con administración de oxitocina, y realizar dicho procedimiento en edades gestacionales superiores a las 39 semanas, ya que se ha observado una disminución notable de morbilidad respiratoria en recién nacidos hasta del $67 \%$ (Abdelazim et al., 2017; Benjamin M. Davis, Glen F. Rall, 2017).

El mayor número de cesáreas electivas se realiza con anestesia raquídea, lo cual no representa un riesgo de morbi-mortalidad fetal, pero su combinación con benzodiacepinas y opioides podría causar estragos maternos como hipotensión, náusea y vómito (Abdollahpour et al., 2015).

\section{CONCLUSIONES}

- De acuerdo a la revisión bibliográfica realizada se concluye que las cesáreas electivas, en especial en el sector privado, son consideradas como las más realizadas en especial en países subdesarrollados como nuestro país con valores superiores al $40 \%$, donde la tasa de natalidad es una de las más altas en todo el mundo.

- A su vez, al tratarse de un proceso quirúrgico que puede traer consigo beneficios tanto para la madre como para el neonato, ocasiona complicaciones, entre las más frecuentes el distrés respiratorio neonatal, el cual representa un riesgo hasta 3 veces mayor comparado al parto por vía vaginal, por lo que se determina como factor de riesgo. En el Ecuador se estimó que existe un riesgo de 2,5 de presentar distrés respiratorio en recién nacidos por cesárea electiva.

- El parto por cesárea programada se realiza en su mayoría en embarazos a término. La entidad más frecuente se describe como la TTRN, patología que se podría prevenir con una prueba de trabajo de parto previo en pacientes con antecedentes de cesárea anterior, administración de oxitocina y corticoides antenatales según protocolos.

\section{REFERENCIAS BIBLIOGRÁFICAS}

Abat \& Wolfman, L. S. B. A. (2015). CARACTERIZACIÓN DE LOS RESULTADOS ADVERSOS MATERNOS Y NEONATALES LUEGO DE UNA OPERACIÓN CESÁREA EN LA E.S.E. CLÍNICA DE MATERNIDAD RAFAEL CALVO SANDRA. Journal of Chemical Information and Modeling, 53(9), 1689-1699. https://doi.org/10.1017/CBO9781107415324.004

Abdelazim, I., Farghali, M. M. M., Elbiaa, A. A. M., Abdelrazak, K. M., Hussain, M., Yehia, A. H., \& Rashad, M. (2017). Impact of antenatal oxytocin infusion on neonatal 
respiratory morbidity associated with elective caesarean section. Archives of Medical Science, 13(3), 629-634. https://doi.org/10.5114/aoms.2017.67292

Abdollahpour, A., Azadi, R., Bandari, R., \& Mirmohammadkhani, M. (2015). Effects of adding midazolam and sufentanil to intrathecal bupivacaine on analgesia quality and postoperative complications in elective cesarean section. Anesthesiology and Pain Medicine, 5(4). https://doi.org/10.5812/aapm.23565

Bautista, L. (2018). Prevalencia de Taquipnea Transitoria Y Factores Asociados en el Área de Neonatología del Hospital Vicente Corral Moscoso, 2015-2016. In Journal of Chemical Information and Modeling (Vol. 53, Issue 9). https://doi.org/10.1017/CBO9781107415324.004

Benjamin M. Davis, Glen F. Rall, M. J. S. (2017). Association of Labor With Neonatal Respiratory Outcomes at 36-40 Weeks of Gestation Beth. Physiology \& Behavior, 176(1), 139-148. https://doi.org/10.1016/j.physbeh.2017.03.040

Benzouina, S., Boubkraoui, M. E. M., Mrabet, M., Chahid, N., Kharbach, A., El-Hassani, A., \& Barkat, A. (2016). Fetal outcome in emergency versus elective cesarean sections at Souissi Maternity Hospital, Rabat, Morocco. The Pan African Medical Journal, 23, 197. https://doi.org/10.11604/pamj.2016.23.197.7401

Bernal, C. (2018). Boletín de Nacimientos por cesárea. In BOLETIN DE NACIMIENTOS POR CESAREA (p. 6). http://www.salud.gob.ec/wpcontent/uploads/2017/01/BOLETÍN-CESAREAS.pdf

C. Garcíal, C. E. C. G. C. C. : situación actual y factores asociados en M. R. S. Q. R. [Internet]. 2018;11(40):28-33. A. from: https://salud. qroo. gob. mx/revista/images/revista40/4. C. S. A. pd. (2018). Cesárea: situación1. C. Garcíal CECGCC: situación actual y factores asociados en Mexico. Revista Salud Quintana Roo, 11(40), 28-33. https://salud.qroo.gob.mx/revista/images/revista40/4. CESÁREA SITUACIÓN ACTUAL.pdf

Carvajal C, J., \& Ralph T, C. (2018). Manual Obstetricia y Ginecología. In Manual de Obstetricia y Ginecología. https://medicina.uc.cl/wpcontent/uploads/2018/08/Manual-Obstetricia-y-Ginecología-2018.pdf

Céspedes, A. (2016). Comparación de la complicacion Sindrome De Insuficiencia Respiratoria Neonatal asociado a Cesarea Electiva, Hospital Rafael Calderon, 20142016. Journal of Chemical Information and Modeling, 53(9), 1689-1699. https://doi.org/10.1017/CBO9781107415324.004

Dileep, A., Khan, N. B., \& Sheikh, S. S. (2015). Comparing neonatal respiratory morbidity in neonates delivered at term by elective Caesarean section with and without 
dexamethasone: Retrospective cohort study. Journal of the Pakistan Medical Association, 65(6), 607-611.

Guia Práctica Clinica. (2016). Recién nacido con dificultad para respirar. 76. https://www.salud.gob.ec/wp-content/uploads/2014/05/GPC-RECIEN-NACIDOCON-DIFICULTAD-PARA-RESPIRAR.pdf

Hernández Núñez, J. (2015). Factores maternos y perinatales influyentes en la morbilidad neonatal. Medwave, 15(6), e6182. https://doi.org/10.5867/medwave.2015.06.6182

Hidalgo, A. (2015). “ Distres Respiratorio , Factores De Riesgos Y Complicaciones En Neonatos Hospitalizados En Ucin " Hospital Universitario De Guayaquil. In Tesis $\begin{array}{llllll}\text { (Vol. } & 1, & \text { Issue } & 1, & \text { p. } & 78) .\end{array}$ http://repositorio.ug.edu.ec/bitstream/redug/36487/1/CD 888- HIDALGO MOLINA ALEX BENJAMIN.pdf

Li, Y., Zhang, C., \& Zhang, D. (2019). Cesarean section and the risk of neonatal respiratory distress syndrome: a meta-analysis. Archives of Gynecology and Obstetrics, 300(3), 503-517. https://doi.org/10.1007/s00404-019-05208-7

Mancilla, J., \& Villanueva, D. (2016). Neonatología. In Insuficiencia Respiratoria Neonatal.

María, E., \& Alonso, G. (2015). Evolución del nacimiento por cesárea: El caso de México Evolution of Caesarean Delivery: The Case of Mexico. ILEMATA Año, 7(18), 2743.

Martínez, A. (2015). Indicaciones y factores asociados al parto por cesárea.

Maternofetal, P. M. (2020). Protocolo: cesárea. CENTRO DE MEDICINA FETAL/NEONATAL DE BARCELONA, 1-26. https://medicinafetalbarcelona.org/protocolos/es/obstetricia/cesarea.pdf

Molina, Y. S. (2019). Diez años de la aplicación del reclutamiento alveolar en pediatría Ten years of alveolar recruitment application in pediatrics. 18(3), 1-17.

Morales Máximo, D. K., \& Morales Máximo, D. K. (2016). Complicaciones materno neonatales de la cesárea electiva versus parto vaginal en pacientes con cesárea previa atendidas en el Instituto Nacional Materno Perinatal, 2015. Repositorio de Tesis UNMSM. http://cybertesis.unmsm.edu.pe/handle/cybertesis/4868

MSP. (2015). Atención del parto por cesárea. https://www.salud.gob.ec/wpcontent/uploads/2014/05/GPC-Atencion_del_Parto_por_cesarea.pdf 
Nakimuli, A., \& Nakubulwa, S. (2015). Incidence and determinants of neonatal morbidity after elective caesarean section at the national referral hospital in Kampala. BMC Research Notes, 8(1), 1-7. https://doi.org/10.1186/s13104-015-1617-7

Petour Gazitúa, F. (2015). Do antenatal corticosteroids in term elective cesarean sections reduce neonatal respiratory morbidity? In Medwave (Vol. 15, Issue 9, p. e6280). Medwave. https://doi.org/10.5867/medwave.2015.09.6280

Pirjani, R. (2018). Elective caesarean section at 36 and 37 weeks compared with outcomes at 38 and 39 weeks. Pediatria Polska, 93(4), 318-324. https://doi.org/10.5114/polp.2018.77997

Rafiei, M., \& Ghare Naz, M. S. (2018). Prevalence, causes, and complications of cesarean delivery in Iran. International Journal of Reproductive BioMedicine, 16(4), 221-234. https://doi.org/10.29252/ijrm.16.4.221

Rosenberg, K. R., \& Trevathan, W. R. (2018). Evolutionary perspectives on cesarean section. Evolution, Medicine and Public Health, 2018(1), 67-81. https://doi.org/10.1093/emph/eoy006

Schmitz, T., Prunet, C., Azria, E., Bohec, C., Bongain, A., Chabanier, P., D’Ercole, C., Deruelle, P., De Tayrac, R., Dreyfus, M., Dupont, C., Gondry, J., Graesslin, O., Kayem, G., Langer, B., Marpeau, L., Morel, O., Parant, O., Perrotin, F., ... Goffinet, F. (2017). Association between Planned Cesarean Delivery and Neonatal Mortality and Morbidity in Twin Pregnancies. Obstetrics and Gynecology, 129(6), 986-995. https://doi.org/10.1097/AOG.0000000000002048

Shraddha K. Shetty, A. K. S. (2015). Association of neonatal respiratory morbidity with timing of elective cesarean delivery. International Journal of Reproduction, Contraception, Obstetrics and Gynecology, 4(2), 292. https://doi.org/10.5455/23201770.ijrcog20150401

Shukla, J., \& Mittal, B. R. (2015). Dimercaptosuccinic acid: A multifunctional cost effective agent for imaging and therapy. Indian Journal of Nuclear Medicine, 30(4), 295-302. https://doi.org/10.4103/0972-3919.164015

Sotiriadis, A., Makrydimas, G., Papatheodorou, S., Jpa, I., \& Mcgoldrick, E. (2018). Corticosteroids for preventing neonatal respiratory morbidity after elective caesarean $\begin{array}{llll}\text { section } & \text { at } & \text { term } & \text { (Review). }\end{array}$ https://doi.org/10.1002/14651858.CD006614.pub3.www.cochranelibrary.com 
Trujillo Angeles, W. A. (2018). Factores de riesgo asociados a taquipnea transitoria del recién nacido en el hospital de ventanilla en el periodo de enero - diciembre del 2016. Tesis, 1(1), $\quad 66 . \quad$ http://repositorio.urp.edu.pe/bitstream/handle/URP/1294/170WTRUJILLO.pdf? sequence $=1 \&$ isAllowed $=\mathrm{y}$

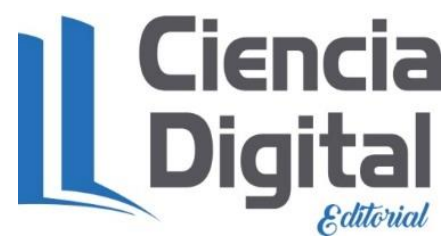




\section{PARA CITAR EL ARTÍCULO INDEXADO.}

Moncayo Rivera, D. M., Moncayo Rivera, C. M., Serpa Calderón, J. M., \& Chacha Suscal , N. R. (2021). Cesárea Electiva como Factor de Riesgo Asociado al Síndrome de Distress Respiratorio Neonatal. Anatomía Digital, 4(1), 225-243. https://doi.org/10.33262/anatomiadigital.v4i1.1561

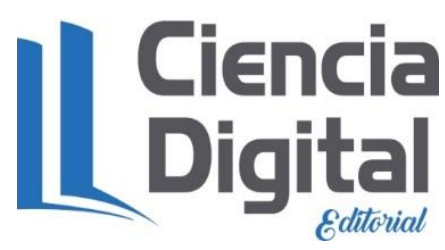

El artículo que se publica es de exclusiva responsabilidad de los autores y no necesariamente reflejan el pensamiento de la Revista Anatomía Digital.

El artículo queda en propiedad de la revista y, por tanto, su publicación parcial y/o total en otro medio tiene que ser autorizado por el director de la Revista Anatomía Digital.
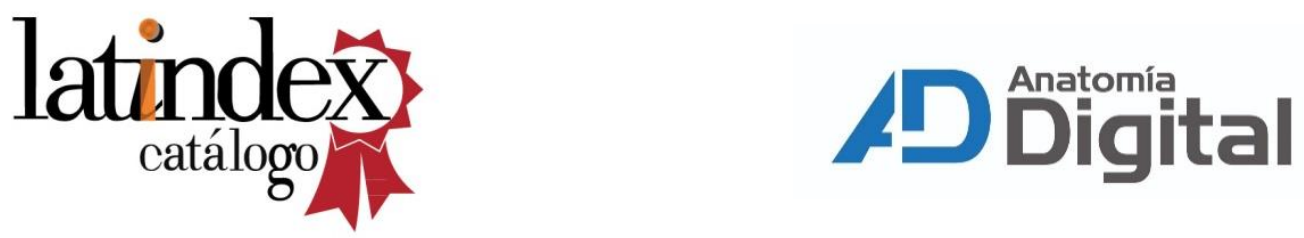